\title{
TELAS DE FÓSFORO PARA PROJEÇÃO DE IMAGEM
}

\author{
Marcos Henrique Mamoru Otsuka Hamanaka, ${ }^{a}$, Michele Odnicki da Silva ${ }^{a}$, Viviane Nogueira Hamanaka ${ }^{\mathrm{b}}$ e Thebano \\ Emílio de Almeida Santos ${ }^{\text {a }}$ \\ aDivisão de Mostradores de Informação, Centro de Tecnologia da Informação Renato Archer, 13069-901 Campinas - SP, Brasil

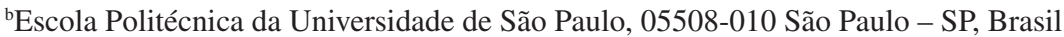

Recebido em 10/01/2017; aceito em 03/04/2017; publicado na web em 13/06/2017

\begin{abstract}
PHOSPHOR SCREENS FOR IMAGE PROJECTION. In this work we describe a methodology to obtain phosphor screens used in various applications to produce images or patterns such as detectors and microscopes. The main advantages of these screens are the particle free and self-heating properties that allows it to be used in controlled environments minimizing the adsorbed gases in the surface of the screen. A polyvinyl alcohol deposited on top of the phosphor layer acts as an adhesive layer preventing the removal of phosphor particles from the screen surface that could result in measurement environment contamination. The tin oxide layer has a high melting point enabling the self-heating. These characteristics on screens are important for ultra-high vacuum environment and to study carbon nanotube emission patterns through field emission microscopy (FEM).
\end{abstract}

Keywords: phosphor screen; image projection; field emission microscopy.

\section{INTRODUÇÃO}

Telas de fósforo são importantes ferramentas empregadas em diversas tecnologias que utilizam íons, elétrons, fótons, radiação ultravioleta (UV) e raios-x, tais como detectores com placas de microcanais (MCPs do inglês micro channel plates), ${ }^{1}$ detectores de raios- $\mathrm{x},{ }^{2}$ detectores de elétrons e $\mathrm{UV},{ }^{3}$ além dos microscópios baseados em emissão de elétrons e sistemas de análises de feixe de elétrons. ${ }^{4}$

Neste artigo será abordada a fabricação e aplicação de telas de fósforo em microscopia por emissão de campo (FEM), que é uma técnica bem estabelecida e tem sido usada desde a década de 50, apresentando imagens, normalmente, com resolução da ordem de angstrons..$^{5-6} \mathrm{~A}$ montagem do sistema é simples, sendo necessário somente o emissor de elétrons, que será estudado, uma grade para propiciar a emissão e uma janela recoberta com fósforo, que é um material que, quando exposto à radiação (feixe de elétrons), emite luz visível.

A tela de fósforo é parte importante do sistema FEM, que utiliza o impacto do feixe de elétrons com certa quantidade de energia para produzir imagens e padrões de emissões. ${ }^{7-11}$ Porém, alguns cuidados devem ser observados, principalmente quanto à resolução da imagem formada, que é dependente da espessura da camada de fósforo. Quanto maior o caminho da radiação dentro da camada de fósforo, maior será a difusão através dos grãos do fósforo, ou seja, pior é a resolução da imagem formada.

A imagem é formada por partículas de luz ou fótons e sua intensidade depende da energia (E) desses fótons, que é diretamente proporcional a sua frequência $\left(\mathrm{v}_{f}\right)$ multiplicada pelo fator de proporcionalidade, ${ }^{12}$ constante de Planck (h), descrito como:

$$
\mathrm{E}=\mathrm{h} v_{f}
$$

Neste trabalho está descrito um método simples para a fabricação de telas de fósforo, por meio da técnica de deposição eletroforética, baseada em suspensão de partículas de fósforo em álcool isopropílico dissolvido em nitrato de magnésio, água deionizada e glicerina. Além disso, uma camada adesiva baseada em álcool polivinílico (PVA) previne que as partículas de fósforo se desprendam da superfície,

*e-mail: mamoru@cti.gov.br permitindo que a tela possa ser utilizada em ambientes controlados como o de ultra alto vácuo ( $10^{-8}$ a $10^{-14}$ Torr), sem gerar contaminantes. A tela de fósforo obtida foi utilizada na caracterização de pontas de tungstênio e nanotubos de carbono por FEM, além de displays de emissão de campo (FED).

\section{PARTE EXPERIMENTAL}

Para a confecção das telas de fósforo foram utilizados substratos de vidro de dimensões de 60 x $110 \mathrm{~mm}$ com $1 \mathrm{~mm}$ de espessura, recobertos com um filme de dióxido de estanho $\left(\mathrm{SnO}_{2}\right)$ como base para a deposição de fósforo por eletroforese. Os filmes de $\mathrm{SnO}_{2}$ têm $700 \mathrm{~nm}$ de espessura, $80 \%$ de transmitância e resistência de folha média de 1000 ohms por quadrado. A Figura 1 mostra as etapas do processo de fabricação da tela de fósforo.

$\mathrm{O}$ substrato de vidro recoberto com $\mathrm{SnO}_{2}$ por deposição química em fase vapor (CVD) é limpo através de imersão do substrato em diferentes soluções (detergente Extran ${ }^{\circledR}(5 \%$ vol) em água deionizada (DI), acetona e álcool isopropílico) com o auxílio de um sistema de banho de ultrassom. Cada banho é realizado por 15 minutos a $70{ }^{\circ} \mathrm{C}$. Por fim, o substrato é seco com jatos de nitrogênio.

Após a limpeza, ocorre a eletrodeposição de níquel (Ni) nas bordas opostas do substrato para obtenção de um bom contato elétrico, pois o filme de $\mathrm{SnO}_{2}$ é resistivo e a resistência entre dois pontos de contato varia de acordo com a distância entre eles. A faixa de contato baseada no níquel melhora e uniformiza o processo de deposição da camada de fósforo.

A solução para a eletrodeposição de Ni contém: 1 litro de água DI; $240 \mathrm{~g}$ de sulfato de níquel hexahidratado; $30 \mathrm{~g}$ de cloreto de níquel hexahidratado; $30 \mathrm{~g}$ de ácido bórico e $2 \mathrm{~g}$ de sacarina sódica. $\mathrm{O}$ ácido bórico foi adicionado ao banho eletroquímico para controle do $\mathrm{pH}$ e a sacarina sódica para proporcionar melhor brilho e uniformidade do Ni. ${ }^{13} \mathrm{O}$ banho é mantido à temperatura ambiente e $\mathrm{pH}$ 4.5. A área do substrato de vidro com filme de $\mathrm{SnO}_{2}$ recoberto com níquel ocupa a faixa da lateral do vidro, tendo a região depositada a largura entre 3 a $5 \mathrm{~cm}$ e o comprimento a dimensão da lateral do vidro. Como contra eletrodo utilizou-se uma placa de níquel. A densidade de corrente da fonte DC é mantida em $3 \mathrm{~A} \mathrm{dm}^{-2}$, durante 1 minuto. Obtém-se um depósito de níquel brilhante e bem homogêneo sobre 
(a)

(b)

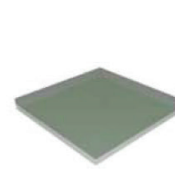

(d)

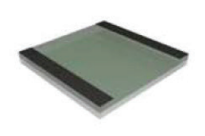

(g)
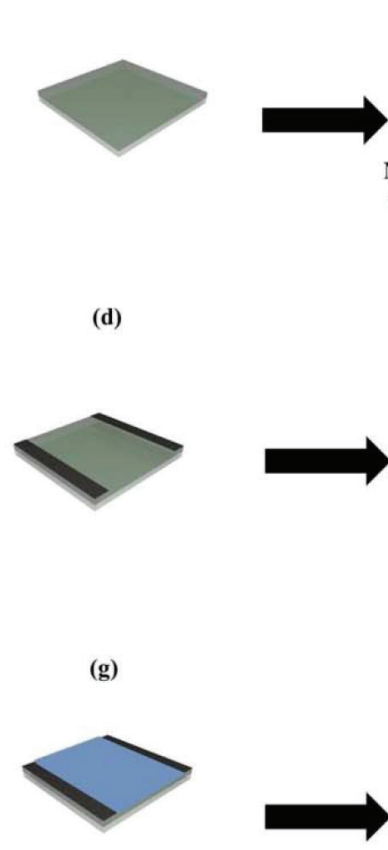

Nível daÁrea exposta

(h)
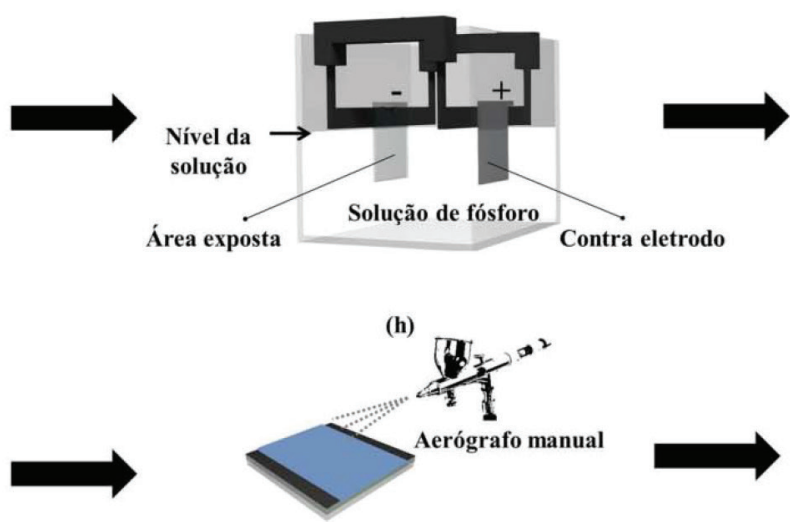

(c)

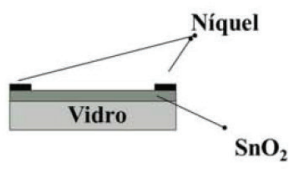

(f)

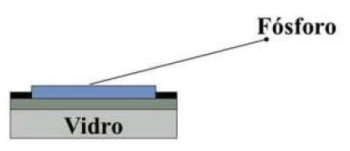

(i)

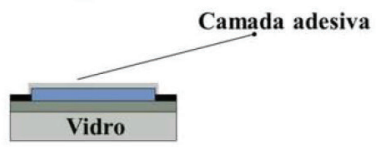

Figura 1. Ilustra o processo de obtenção da tela de fósforo: a) Substrato de vidro recoberto com $\mathrm{SnO}_{2}$; b) eletrodeposição de níqueld) substrato de vidro recoberto com $\mathrm{SnO}_{2}$ e níquel; e) deposição eletroforética; $\mathrm{g}$ ) substrato de vidro recoberto com $\mathrm{SnO}_{2}$, níquel e fósforo; $h$ ) deposição da camada adesiva por spray; c), f) e i) apresentam o perfil das camadas depositadas

toda a superfície submersa e exposta do substrato de $\mathrm{SnO}_{2}$. A reação básica do processo é:

$$
\mathrm{Ni}^{2+}+2 \mathrm{e}^{-} \rightarrow \mathrm{Ni}_{(\mathrm{S})}
$$

Com as bordas recobertas com níquel inicia-se a deposição eletroforética do fósforo. A solução utilizada para a deposição é baseada em $1 \mathrm{~L}$ de álcool isopropílico, $4 \mathrm{~g}$ de fósforo $\mathrm{ZnS}: \mathrm{Ag}$ (Luminophor), $0,26 \mathrm{~g}$ de nitrato de magnésio hexa-hidratado, $10 \mathrm{~mL}$ de água deionizada e $10 \mathrm{~mL}$ de glicerina. ${ }^{14-15}$ Durante a deposição eletroforética o $\mathrm{Mg}\left(\mathrm{NO}_{3}\right)^{+}$é o cátion predominante disponível para carregar e formar camada de aderência do fósforo. As reações do processo são: ${ }^{13,16,17}$

$$
\begin{gathered}
2 \mathrm{H}_{2} \mathrm{O}+2 \mathrm{e}^{-} \rightarrow \mathrm{H}_{2(\mathrm{~g})}+2 \mathrm{OH}^{-} \\
\mathrm{Mg}\left(\mathrm{NO}_{3}\right)^{+}+2 \mathrm{OH}^{-} \rightarrow \mathrm{Mg}(\mathrm{OH})_{2}+\mathrm{NO}_{3}{ }^{-} \\
\mathrm{Mg}^{2+}+2 \mathrm{C}_{3} \mathrm{H}_{7} \mathrm{OH} \rightarrow \mathrm{Mg}\left(\mathrm{C}_{3} \mathrm{H}_{7} \mathrm{O}\right)_{2}+2 \mathrm{H}^{+} \\
\mathrm{Mg}\left(\mathrm{C}_{3} \mathrm{H}_{7} \mathrm{O}\right)_{2}+2 \mathrm{H}_{2} \mathrm{O} \rightarrow \mathrm{Mg}(\mathrm{OH})_{2}+2 \mathrm{C}_{3} \mathrm{H}_{7} \mathrm{OH}
\end{gathered}
$$

A deposição é controlada pelo potencial que é mantido em 25 VDC durante 40 minutos. Após a deposição da camada de fósforo o substrato é deixado na estufa à $120^{\circ} \mathrm{C}$ por $18 \mathrm{~h}$ para completa secagem. A camada de fósforo depositada é de cor branca e apresenta boa uniformidade sobre toda a superfície do filme de $\mathrm{SnO}_{2}$, porem com uma baixa aderência ao substrato de vidro, podendo ser facilmente removida e contaminar a câmara de vácuo do sistema de caracterização. Para solucionar este problema foi depositada uma camada adesiva sobre o fósforo.

A camada adesiva foi estabelecida nas indústrias que produziam telas com fósforos e resulta numa melhora significativa na adesão entre as partículas de fósforo e o substrato de vidro. ${ }^{18}$ Neste processo é empregado o álcool polivinílico (PVA) $\left(2 \mathrm{~g} \mathrm{~L}^{-1}\right)$ e o dicromato de amônio (ADC) (0.01 - $\left.0.02 \mathrm{~g} \mathrm{~L}^{-1}\right)$ diluídos em água DI, formando assim o material adesivo, que é depositado sobre o fósforo utilizando um aerógrafo manual. ${ }^{18}$ Parte da solução aquosa penetra entre as partículas de fósforo, revestindo-as com uma película bem fina, permitindo que o adesivo desempenhe um papel de reforço da força adesiva entre as partículas fosforescentes e a camada de óxido. Após a deposição, realiza-se a cura do material adesivo por meio da radiação ultravioleta (UV $)^{18}$ $(\lambda=365 \mathrm{~nm})$ com densidade de potência de $10 \mathrm{~mW} / \mathrm{cm}^{2}$ durante 2 minutos. A luz UV provoca uma reação fotoquímica induzindo ligação cruzada entre as cadeias quebradas de PVA e os íons de Cr.

O controle do processo de fabricação foi realizado com o auxílio de equipamentos que permitiram observar as características dos materiais em cada procedimento descrito neste trabalho.

As espessuras das camadas de $\mathrm{SnO}_{2}$ e Ni foram obtidas com o equipamento DEKTAK modelo 3030. Para o fósforo foi utilizado perfilômetro Zygo modelo NewView 5000. A transmitância do filme de $\mathrm{SnO}_{2}$ foi medida com o espectrofotômetro da PerkinElmer modelo Lambda 900. A resistência de folha dos filmes de $\mathrm{SnO}_{2}$ foi obtida pela técnica de quatro pontas com equipamento da Thin Film Devices Inc modelo FPP-2000.

A rugosidade e análises superficiais do fósforo foram obtidas com a microscopia de força atômica (AFM) da Nanosurf modelo EasyScan. O teste de adesão foi realizado baseado na norma ISO 2819 item 2.4 (Peel test) de acordo com o procedimento da fita adesiva descrito no último parágrafo. ${ }^{19}$

A temperatura foi monitorada com o medidor de infravermelho da Minolta modelo Cyclo 33CF.

\section{RESULTADOS E DISCUSSÃO}

A adição de sacarina sódica no processo de eletrodeposição de níquel para obtenção dos eletrodos de contato resultou em um filme mais uniforme sem manchas pretas, com um brilho intenso, boa aderência ao filme fino de dióxido de estanho e espessura média de $450 \mathrm{~nm}$.

A Figura 2 a apresenta uma imagem da tela de fósforo obtida 


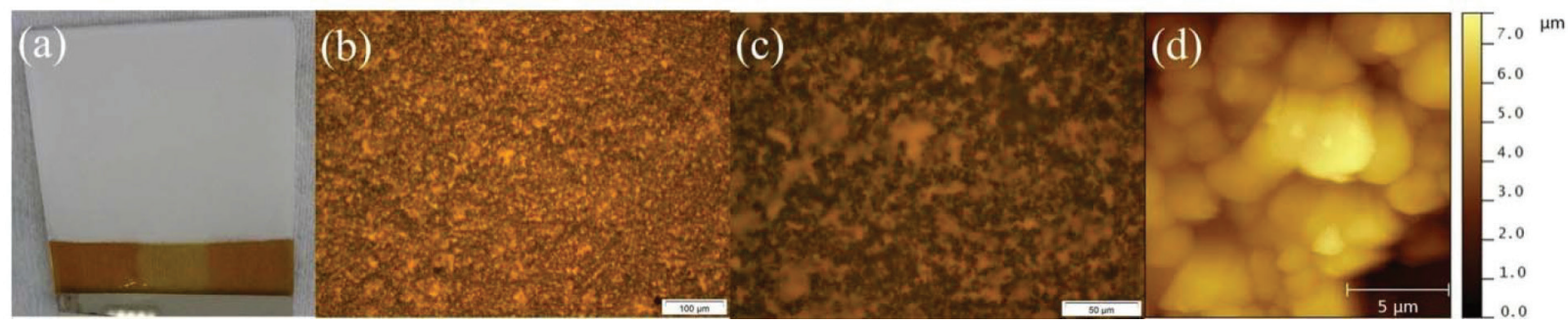

Figura 2. Imagens da tela de fósforo azul com dimensões de $70 \mathrm{~mm} \times 50 \mathrm{~mm} \times 1,2 \mathrm{~mm}$ : a) foto da tela de fósforo; b) e c) imagens da microscopia óptica onde se observam pontos com maior concentração de partículas de fósforo e d) imagem de microscopia de força atômica

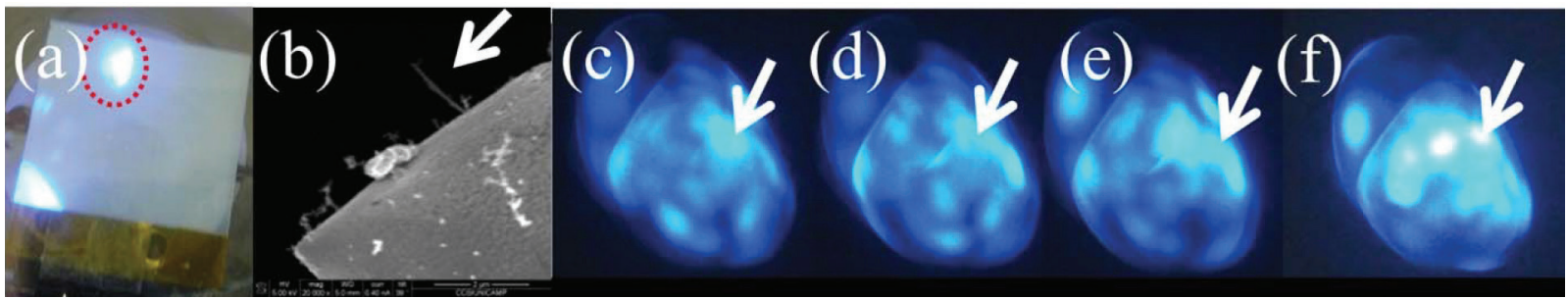

Figura 3. Imagens do sistema FEM: a) tela de fósforo projetando imagens da emissão de elétrons dos nanotubos de carbono; b) microscopia eletrônica de varredura (MEV) do CNT que está emitindo; (c) a (f) detalhes dos pontos de adsorção de gases que se movimentam durante a aquisição das imagens por FEM. A tensão utilizada para emissão foi de aproximadamente 800VDC

utilizando a metodologia descrita anteriormente. As camadas de fósforo (com espessura de $23 \mathrm{~mm}$ ) e de PVA (com espessura de 250 $\mathrm{nm}$ ) apresentaram boa uniformidade de deposição. As Figuras $2 \mathrm{~b}$ e $2 \mathrm{c}$ apresentam imagens de microscopia óptica nas quais observa-se que existem pontos com maior concentração de partículas de fósforo, apesar do filme apresentar boa uniformidade de deposição. A presença da camada adesiva somente é observada devido à coloração levemente amarelada que recobre toda a superfície da tela após sua aplicação e pela diminuição da rugosidade para 1,3 $\mu \mathrm{m}$, Figura $2 \mathrm{~d}$. Antes da aplicação de PVA não foi possível obter o valor da rugosidade pela técnica de microscopia de força atômica (AFM).

Normalmente o fósforo apresenta baixa aderência superficial, sendo necessário adicionar algum elemento para melhor a aderência e em alguns casos tratamento térmico em temperaturas elevadas. ${ }^{16-18}$ Neste trabalho a solução adesiva utilizando PVA e ADC melhorou a aderência do fósforo e diminui a rugosidade superficial da tela, sem a necessidade de tratamento térmico. ${ }^{18} \mathrm{~A}$ adesão da camada de fósforo e da camada de PVA foram avaliadas qualitativamente por meio de uma fita adesiva, seguindo a norma ISO 2819. A camada de fósforo sem PVA foi removida facilmente, enquanto para a camada protegida com PVA não se observou a remoção do fósforo.

A tela de fósforo foi montada em um sistema de microscopia por emissão de campo (FEM) que foi projetado e construído para estudar as fontes emissoras de elétrons. Este sistema é composto pelo emissor de elétrons, que será estudado e está aterrado; uma grade, que será polarizada com tensão contínua, para propiciar a emissão; e uma janela recoberta com fósforo, que emite luz visível, formando a imagem do emissor ao ser atingida pelos elétrons emitidos.

As pontas emissoras são posicionadas no porta-pontas, que permite o ajuste da distância à grade de fios de aço (mesh $=40 \mu \mathrm{m})$. Em geral, a distância entre o emissor e grade é de $0,1 \mathrm{~cm}$.

A utilização da tela de fósforo no sistema FEM possibilitou o estudo de nanotubos de carbono (CNTs) através da projeção das imagens decorrentes do impacto dos elétrons gerados pelo campo elétrico do sistema, como mostra a Figura 3a. As imagens permitem avaliar diversas características dos CNTs, como estrutura das extremidades, se os CNTs possuem tampas, estimar a área de emissão, verificar a quantidade de nanotubos que estão emitindo, comparar diferentes emissores e estudar seu comportamento de emissão.
As Figuras de 3c a $3 \mathrm{f}$ mostram pontos com brilho mais intenso, que correspondem a gases adsorvidos na superfície do CNT. Estes pontos se movem e se tornam menos intensos ao longo do tempo.

A utilização do filme de oxido de estanho permitiu diminuir a quantidade de gases adsorvidos devido à característica de o filme operar como um elemento aquecedor através da aplicação de uma diferença de potencial entre dois eletrodos de níquel, situados em lados opostos da tela de fósforo. A temperatura máxima depende: da espessura do filme de oxido de estanho, sendo os melhores resultados para espessuras entre 200 a $800 \mathrm{~nm}$; da velocidade de aquecimento e resfriamento; e das características intrínsecas de cada material, descritas na tabela $1 .{ }^{20}$ Temperaturas acima de $100{ }^{\circ} \mathrm{C}$ alteraram a coloração da tela de fósforo, intensificando a cor amarela.

Tabela 1. Propriedades mecânicas de vidros soda lime e filmes de óxido de estanho

\begin{tabular}{lcc}
\hline & $\begin{array}{c}\text { Vidro } \\
\text { comum }\end{array}$ & $\begin{array}{c}\text { Óxido de } \\
\text { estanho }\end{array}$ \\
\hline Módulo de Young ou módulo de elasticidade $(\mathrm{GPa})$ & 72 & 263 \\
Razão de Poisson & 0,23 & 0,29 \\
Coeficiente de expansão linear $\left(10^{-6} / \mathrm{K}\right)$ & 8,3 & 4,13 \\
Stress térmico do filme de $\mathrm{SnO}_{2}(\mathrm{GPa})$ & $-0,63$ & n.d. \\
\hline
\end{tabular}

\section{CONCLUSÃO}

Este trabalho apresentou um método simples para a fabricação de telas de fósforo, por meio de deposição eletroforética de uma suspensão de partículas de fósforo em álcool isopropílico dissolvido em nitrato de magnésio, água deionizada e glicerina. A tela de fósforo com a camada de PVA apresentou boa aderência ao substrato, evitando que as partículas de fósforos fossem liberadas facilmente por atrito mecânico e diminuindo a rugosidade superficial. A tela foi utilizada no sistema de microscopia por campo elétrico e viabilizou o estudo da emissão de elétrons de nanotubos de carbono. O filme de dióxido de estanho permitiu aquecer a tela de fósforo, contribuindo com a diminuição dos gases adsorvidos em sua superfície e melhorando as imagens e padrões dos emissores. 


\section{AGRADECIMENTOS}

Agradecemos ao V. L. Pimentel que realizou as medidas de AFM, ao A. B Benetti pelas ilustrações deste trabalho, ao I. A. Pereira que forneceu os substratos recobertos com o $\mathrm{SnO}_{2}$, ao Dr. D. den Engelsen, que forneceu o fósforo, ao J. Peressinotto que desenvolveu a parte mecânica do sistema FEM e ao Laboratório Nacional de Nanotecnologia (LNNano), que disponibilizou a infraestrutura para a medida de rugosidade por microscopia por força atômica.

\section{REFERÊNCIAS}

1. Nakamura, A.; Shimojima, T.; Nakano, M.; Iwasa, Y.; Ishizaka, K.; Struct Dyn. 2016, 3, 064501.

2. Miura, E.; Kuroda, R.; Toyokawa, H.; J. Phys. 2016, 688, 1.

3. Gauthier, M.; Goede, S.; Schumaker, W.; Roedel, C.; Galtier, E. and Glenzer, S. H.; SLAC Report-R-1061, 2015.

4. Bok, J.; Horáček, M.; Kolařík, V.; Urbánek, M.; Matějka, M.; Krzyžánek, V.; Microelectron. Eng. 2016, 149, 117.

5. Dean, K. A.; Chalamala, B. R.; J. Appl. Phys. 1999, 85, 3832.

6. Hata, K.; Takakura, A.; Saito, Y.; Surf. Sci. 2001, 490, 296.

7. Bonard, J. M.; Maier, F.; Stockli, T.; Châtelain, A.; De Heer, W. A.; Salvetat, J. P.; Forró, L.; Ultramicroscopy 1998, 73, 9.
8. Bonard, J. M.; Salvetat, J. P.; Stöckli, T.; Forró, L.; Châtelain, A.; Appl. Phys. A: Mater. Sci. Process. 1999, 69, 245.

9. Bonard, J. M.; Kind, H.; Stockli, T. and Nilsson, L. O.; Solid-State Electron. 2001, 45, 893.

10. De Jonge, N.; Allioux, M.; Doytcheva, M.; Kaiser, M.; Teo, K. B.; Lacerda, R. G and Milne, W. I.; Appl. Phys. Lett. 2004, 85, 1607.

11. Saito, Y.; Hamaguchi, K.; Nishimo, T.; Hata, K.; Tohji, K.; Ksuya, A. and Nishina, Y.; Jpn. J. Appl. Phys. 1997, 36, 1340.

12. Hewitt, P. G.; Fisica Conceitual, 9a ed., Artmed: São Paulo, 2002, cap. 30 .

13. Rose, I.; Whittington, C.; Nickel Plating Handbook, OMG group, 2002.

14. Talbot, J. B.; Sluzky, E.; Kurinec, S. K.; J. Mater. Sci. 2004, 39, 771.

15. Kang, S. W.; Yoo, J. S.; Lee, J. D.; J. Vac. Sci. Technol., B: Nanotechnol. Microelectron.: Mater, Process., Meas., Phenom. 1998, 16, 2891.

16. Zhanga, C.; Uchikoshi, T.; Kitabatake, T.; Sakka, Y.; Hirosaki, N.; Appl. Surf. Sci. 2013, 280, 229.

17. Besra, L.; Liu, M.; Prog. Mater. Sci. 2007, 52, 1.

18. Yum, J.; Choi, K.; Sung, Y.; J. Electrochem. Soc. 2003, 150, H43.

19. ISO 2819, Metallic coatings on metallic substrates - Electrodeposited and chemically deposited coatings - Review of methods available for testing adhesion, Switzerland, 1980.

20. Green, D. J.; Glass Technol. 2000, 41, 1. 